\title{
Detailed characterization of the posttranscriptional gene-silencing-related small RNA in a GUS gene-silenced tobacco
}

\author{
GYÖRGY HUTVÁGNER, ${ }^{1,2}$ LUDMILA MLYNÁROVÁ, ${ }^{1,3}$ and JAN-PETER NAP ${ }^{1}$ \\ ${ }_{1}^{1} \mathrm{BU}$ Genomics, Plant Research International, Wageningen, The Netherlands \\ ${ }^{2}$ Agricultural Biotechnology Center, Gödöllö, Hungary \\ ${ }^{3}$ Institute of Plant Genetics and Biotechnology, Nitra, Slovak Republic
}

\begin{abstract}
Posttranscriptional gene-silencing phenomena such as cosuppression and RNA interference are associated with the occurrence of small, about 21-23 nt short RNA species homologous to the silenced gene. We here show that the small RNA present in silenced transgenic plants can easily be detected in total RNA isolated according to standard procedures. This will allow for the development of routine and early screenings for the presence of small RNA species and, therefore, gene silencing in transgenic plants. We further demonstrate that the small RNA fraction can be visualized with the SYBR Green II RNA stain, isolated from a gel, labeled and used as a specific probe. Using these approaches, we have fine-mapped the sequences of the GUS gene that are represented in the small RNA fraction of a GUS-silenced tobacco line containing an inverted repeat of the GUS gene. In this tobacco line, the silencingassociated small RNA is a mixture of fragments that cover the $3^{\prime}$ two-thirds of the GUS coding region. The $5^{\prime}$ coding and the $3^{\prime}$ noncoding ends of the GUS mRNA are not represented in the small RNA fraction. The RNA fragments are not likely to be a primary synthesis product of an RNA-dependent RNA polymerase, but rather degradation products from nuclease activity. Surprisingly, RNA isolated from wild-type, untransformed plants showed the presence of a similar-sized small RNA pool. This might indicate the existence of small RNA species from putative endogenous genes that are down regulated by a similar posttranscriptional gene-silencing mechanism. The possibility of isolating and labeling the small RNA pool from wild-type plants will provide a way to identify and study such putative genes.
\end{abstract}

Keywords: cosuppression; posttranscriptional gene silencing; RNA labeling; SYBR Green II stain

\section{INTRODUCTION}

Recently, small RNA species have been shown to be associated with posttranscriptional gene silencing (PTGS) in tobacco, tomato, and Arabidopsis plants (Hamilton \& Baulcombe, 1999; Dalmay et al., 2000a). In cases of both transgene-induced and virus-induced PTGS it was demonstrated that plants in the silenced state contained an approximately 21-23 nt antisense RNA species. PTGS in plants results in a severe reduction of the amount of steady-state RNA in the cytoplasm, whereas transcriptional activity in the nucleus is generally normal. In other organisms, similar phenomena occur, such as quelling in the fungus Neurospora (Cogoni \& Macino, 1997). In addition, double-stranded RNA was demonstrated to be a potent mediator of

Reprint requests to: Jan-Peter Nap, BU Genomics, Plant Research International, P.O. Box 16, NL-6700 AA Wageningen, The Netherlands; e-mail: J.P.H.Nap@plant.wag-ur.nl. sequence-specific gene silencing known as RNA interference or RNAi (Fire et al., 1998; for a review, see also Sharp, 1999) in also a posttranscriptional manner. This was shown in Caenorhabditis elegans (Fire et al., 1998; Montgomery et al., 1998), during early development in mouse (Wianny \& Zernicka-Goetz, 2000), and during flower development in Arabidopsis thaliana (Chuang \& Meyerowitz, 2000). Also in a range of invertebrate species including Drosophila (Misquitta \& Patterson, 1999), trypanosomes (Ngo et al., 1998), and planaria (SanchezAlvorado \& Newmark, 1999) dsRNA and RNAi are effective. For a recent review, see Bosher and Labouesse (2000). Moreover, in a Drosophila in vitro system, RNA segments of 21-23 nt were generated upon introduction of dsRNA (Zamore et al., 2000). A similar small RNA cofractionated with the enzyme fraction that degraded exogenous transcripts homologous to the double-stranded RNA used for transfection (Hammond et al., 2000). In C. elegans, cosuppression and RNAi were recently shown to be linked genetically (Ketting \& 
Plasterk, 2000). RNAi in C. elegans, quelling in Neurospora, and PTGS in Arabidopsis require similar genes (Dalmay et al., 2000b; Mourrain et al., 2000). These data link RNAi, cosuppression, and small RNA species. Small RNA species-mediated PTGS may therefore be a conserved and widespread method of regulation of gene activity.

PTGS only affects sufficiently homologous sequences and has been proposed to be a natural defense system against viruses and/or transposons. The capacity of PTGS to specifically degrade homologous RNA is currently used for functional genomics in plants (Baulcombe, 1999). Various models for the mechanism of PTGS in plants (Lindbo et al., 1993; Baulcombe \& English, 1996) postulated the involvement of an "aberrant RNA" to account for the apparent sequence specificity of PTGS. This undefined RNA could be produced by activity of an RNA-dependent RNA polymerase (RdRp; Wassenegger \& Pélissier, 1998). By mutagenesis, the Arabidopsis RdRp gene was shown to be required for PTGS (Dalmay et al., 2000b; Mourrain et al., 2000). The small RNA species identified by Hamilton and Baulcombe (1999) may very well represent the sequence specificity determinant of the PTGS regulatory system. Detailed analyses of the characteristics of the small RNA will therefore contribute to the further mechanistic understanding of PTGS.

To visualize the novel small RNA species, Hamilton and Baulcombe (1999) enriched the RNA of silenced and active plants for low-molecular-weight molecules by first removing the high-molecular-weight RNA species with polyethylene-glycol/salt precipitation or filtration, followed by ion-exchange chromatography. The enriched RNA fraction was separated on polyacrylamide/urea gels, blotted, and the small RNA was subsequently visualized by low-stringency hybridization with RNA probes. We here show that the elaborate procedure to enrich for the PTGS-associated small RNA spe- cies is not required. A total RNA isolation is sufficient, resulting in a faster, simpler, and equally reproducible visualization of the presence of PTGS-associated small RNA in silenced plants. Moreover, we show that the small RNA species can be isolated from a gel, labeled, and used as a probe. Both methods were used to characterize the features of the small RNA species in a GUS-silenced tobacco plant. The analyses show that all but the first $554( \pm 15)$ nt of the GUS coding region give rise to small RNA molecules. To our surprise, the methods of analysis developed here allowed us to demonstrate similar-sized small RNAs in untransformed, wild-type tobacco and Arabidopsis plants. This might imply the existence of small RNA from endogenous genes in plants that are down regulated by the same posttranscriptional gene-silencing mechanism, but do not require the introduction of homologous transgenes. For conclusive evidence of the existence of such endogenous genes, they should be isolated. The possibility of isolating and labeling the small RNA pool from wild-type plants will provide a way to identify and study such putative genes.

\section{RESULTS}

\section{Visualization of PTGS-associated RNA in total RNA of silenced tobacco}

Six-to-eight-week-old greenhouse-grown NLG4-He plants were used for RNA analyses. As controls, wildtype tobacco and the GUS-active tobacco line ANLGA13 were used. The T-DNA configurations in the NLG4-He and ANGLA13 plants are shown in Figure 1. Extensive Southern blot analyses showed that the GUS-active tobacco ANGLA13 (in hemizygous state) contains a single copy of the incoming T-DNA, whereas tobacco NLG4-He contains a single but complex locus. This locus consists of a complete inverted repeat of the

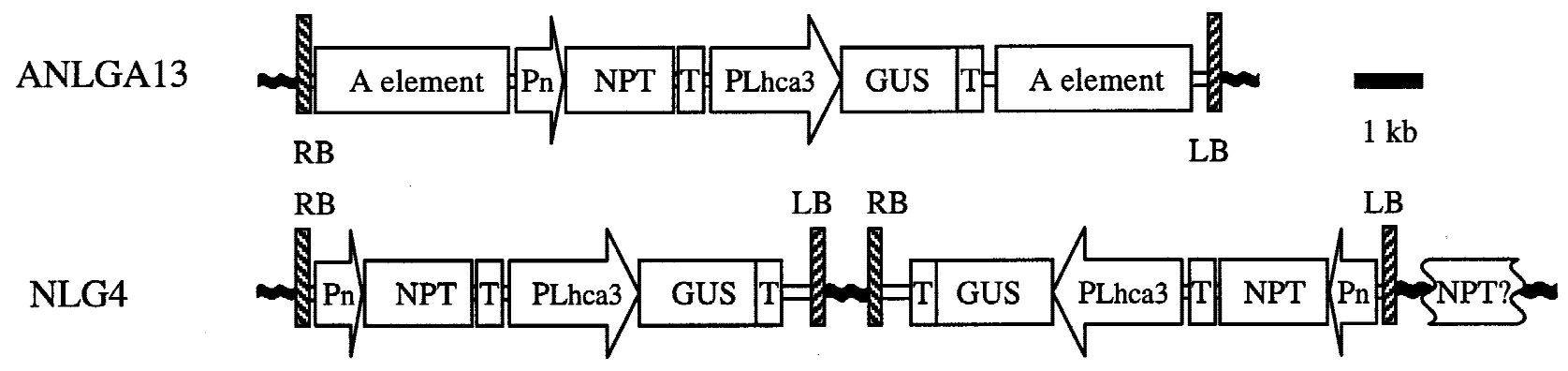

FIGURE 1. T-DNA configuration in the transgenic tobacco lines used for analysis. Tobacco line ANGLA13 contains, in hemizygous state, a single copy of the T-DNA from plasmid pANGLA. Tobacco line NLG4 contains a complex single locus of the T-DNA from plasmid NLG, consisting of a full inverted repeat interrupted by about $500 \mathrm{bp}$ of genomic DNA, plus an additional truncated NPTII fragment that has not been mapped in detail. In both DNAs, the GUS gene is driven by the light-regulated potato Lhca3.St.1 promoter (PLhca3). The selectable marker is the neomycin phosphotransferase II gene (NPT) driven by the nopaline synthase (nos) promoter (Pn). Both genes also carry the nos terminator sequence (T). The ANGLA13 DNA contains, in addition, the chicken lysozyme matrix-associated region (known and indicated as A element; Mlynárová et al., 1994) around the GUS and NPTII transgenes. The right and left border of the Agrobacterium T-DNA are indicated by RB and LB, respectively. 
incoming T-DNA, interrupted by a stretch of about $500 \mathrm{bp}$ genomic DNA, in addition to a third truncated NPTII gene. In both the homozygous (NLG4-Ho) and hemizygous (NLG4-He) states, the NLG4 locus itself has very low GUS activity in leaves. Run-off analyses in NLG4 nuclei has confirmed that this silencing is posttranscriptional (our unpubl. results). When crossed to transgenic tobacco lines with an active GUS gene, the NLG4 locus behaves as a dominant silencer over unlinked, active GUS alleles that are identical in sequence (Nap et al., 1997).

The phenol-guanidine-isothiocyanate-based RNA isolation using the TriZOL reagens (Life Technologies) is a fast and convenient method to isolate intact total RNA virtually free of DNA from, among many others, tobacco and Arabidopsis plants. The standard protocol used involves a single isopropanol precipitation (see Material and Methods for details). Different amounts of total RNA were separated on $6 \%$ polyacrylamide/8 M urea gels, electroblotted to Hybond $\mathrm{N}+$ (Amersham), fixed, and hybridized at $50^{\circ} \mathrm{C}$ to radioactively labeled GUS DNA. Figure 1 shows the phosphor image of such an RNA blot. In $5 \mu \mathrm{g}$ of total RNA isolated from NLG4$\mathrm{He}$, a small RNA homologous to the GUS probe is easily detectable. No such small RNA species was ever detectable in as much as $50 \mu \mathrm{g}$ RNA of either wild-type tobacco or the GUS-active tobacco line ANLGA13 (Fig. 2). We routinely could detect silenced transgenehomologous small RNA in total RNA from various silenced plants, including Arabidopsis (data not shown). This establishes that the TriZol protocol is sufficient to visualize the small RNA species and omits the need for elaborate enriching of the small RNA fraction. To esti-

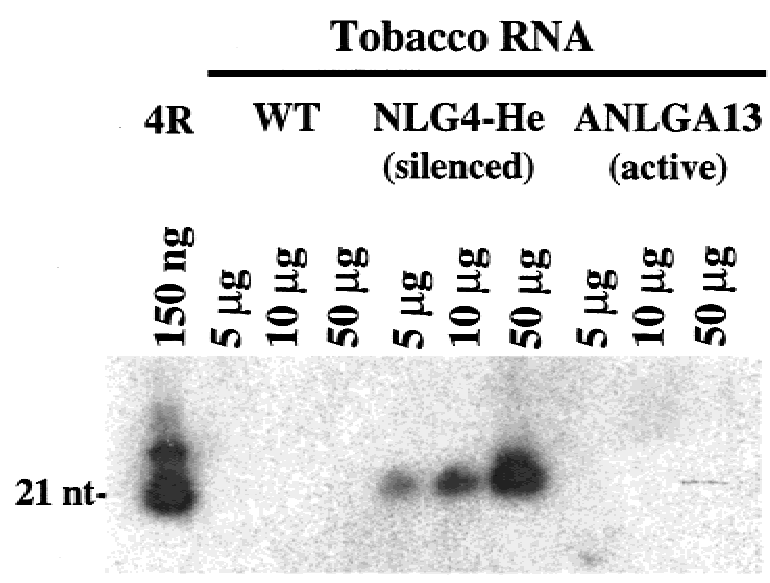

FIGURE 2. Detection of GUS gene-homologous small RNA in GUSsilenced tobacco. Phosphor image of an RNA blot containing increasing amounts of total RNA isolated from leaves, hybridized with the full-length GUS sequence. 4R is a 21-nt oligomer from the GUS sequence that is used as both size marker and positive control for hybridization. WT: RNA from wild-type tobacco; NLG4-He: RNA from the GUS-silenced, hemizygous transgenic line NLG4-He (see Fig. 1); ANGLA13: RNA from the GUS-active, homozygous transgenic line ANGLA13 (see Fig. 1). mate the size of the hybridizing RNA species, a 21-nt GUS oligomer is used as hybridization control. The hybridizing small RNA species is a bit larger than this 21-nt oligomer. With the use of RNA markers for the size estimation it is likely that the small RNA would have been estimated to be 21-23 nt in length (Hamilton \& Baulcombe, 2000), as reported for Drosophila (Zamore et al., 2000). On the basis of hybridization intensity, the GUS-homologous small RNA species is quite abundant in the total RNA isolate.

\section{Characterization of the GUS-gene-derived small RNA species in a silenced tobacco}

The relatively easy detection of the GUS-gene-derived small RNA species in tobacco line NLG4-He facilitates further characterization and analyses of this small RNA. First, we analyzed from what part or parts of the GUS gene the small RNA sequence is derived. Five fragments (GUS1-GUS5) spanning the entire GUS coding sequence were isolated with PCR, cloned in pGEM-T Easy (Promega), and used as probes to detect GUS sequence-derived small RNA in RNA from NLG4-He and ANGLA13. In Figure 3A, the relative positions of the GUS subfragments are given. Fragment GUS1 (295 bp) starts from the ATG of the GUS coding sequence. GUS5 (274 bp) contains the $3^{\prime}$ nontranslated region of the GUS gene. The hybridization results are given in Figure 3B. In all cases, one of the oligomers used to generate the PCR product was used as positive hybridization control. Small RNA was detected with the GUS2, GUS3, and GUS4 subfragments of the GUS sequence as probes, a 1,496-bp sequence. This establishes that the GUS-derived small RNA in tobacco NLG4-He is not a single RNA species, but a mixture of similar-sized RNA molecules of different sequence composition. No small RNA could be detected with the GUS1 and GUS5 probes. This indicates that in tobacco NLG4-He the $5^{\prime}$ coding region and the $3^{\prime}$ noncoding region of the GUS mRNA are not generating small RNA molecules or are generating them much less.

\section{Isolation and labeling of the small RNA fraction}

The amount of GUS-derived small RNA in the silenced NLG4-He tobacco line is relatively abundant. This suggested the possibility of isolating this fraction of small RNA molecules from the gel and labeling it with radioactivity. With oligomers of 21 and $35 \mathrm{nt}$ stained with SYBR Green II RNA stain (Molecular Probes) as size markers, the desired region of small RNA could be identified in a $17 \%$ polyacrylamide/urea gel, cut from the gel, and isolated with a crush and soak method (Faulkner-Jones, 1995). The isolated RNA was subsequently labeled with $\gamma^{-32}$ P-ATP (ICN) using the forward reaction of the Kinase Max kit (Ambion). Under these reaction conditions, no labeling was obtained for 


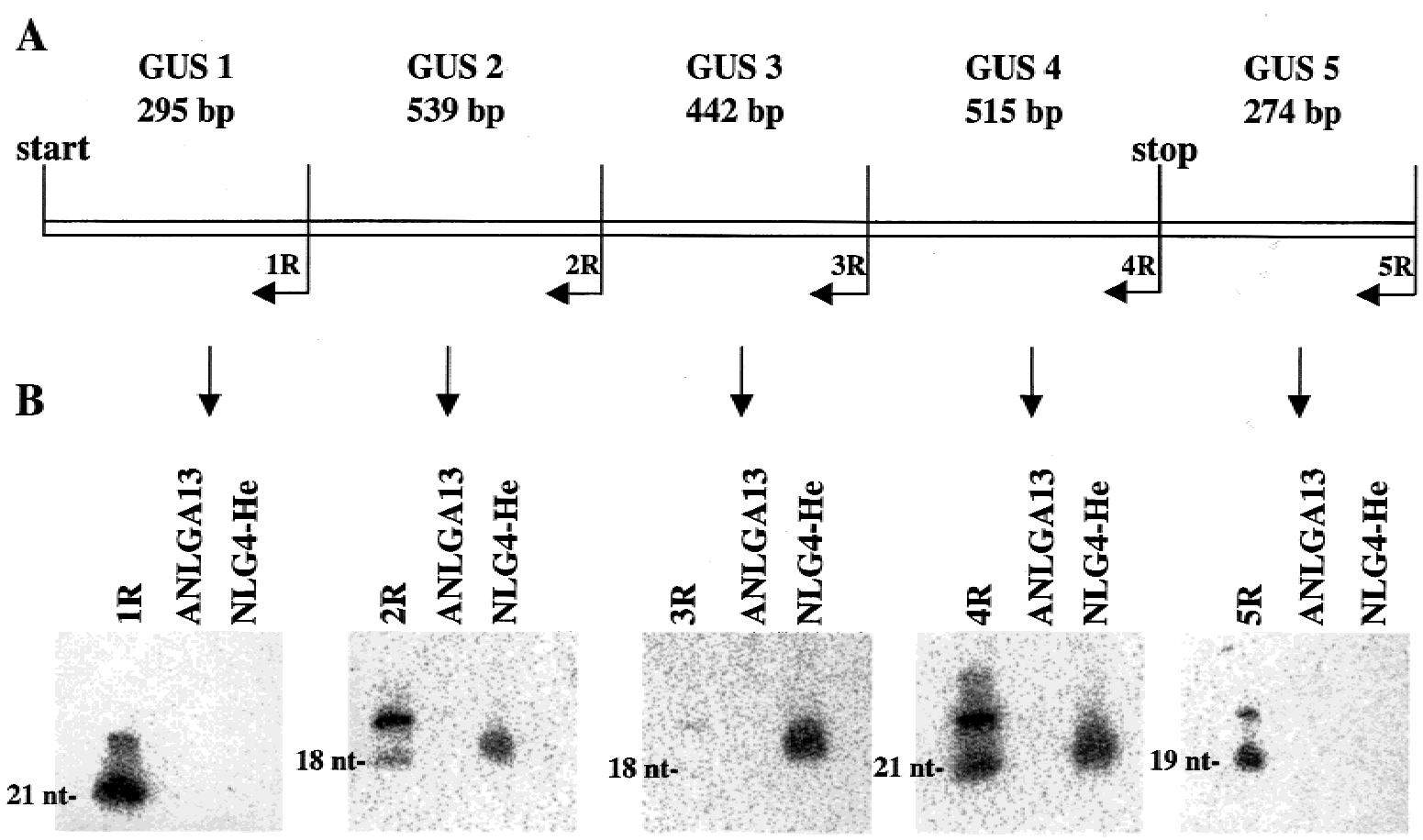

FIGURE 3. Mapping of the GUS regions represented in the small RNA fraction using GUS subclone DNA as a probe. A: Position and size of the GUS subclones in the full-length GUS sequence. The arrows indicate the positions of the reverse primers used. B: Phosphor image of RNA blots containing $30 \mu \mathrm{g}$ of total RNA isolated from leaves, hybridized with radioactively labeled GUS subclones. $1 \mathrm{R}-5 \mathrm{R}$ are the primers used as the size marker and positive control for hybridization. For each, $150 \mathrm{ng}$ was loaded. Indication of plant lines is as in Figure 2.

in vitro prepared, 5'-phosphated RNA (data not shown). Analysis of a dilution of the labeled RNA on a gel revealed a discrete band with a size between 20 and $30 \mathrm{bp}$, indicating successful labeling of small RNA (Fig. 4). The labeled small RNA obtained from NLG4-He tobacco was subsequently used as a probe on a blot

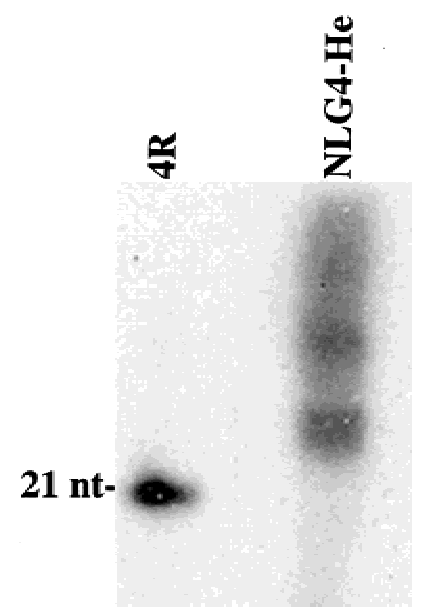

FIGURE 4. Labeling of gel-purified small RNA. Phosphor image of a dried polyacrylamide/urea gel containing appropriate amounts of a $\gamma^{-}{ }^{33}$ P-ATP-labeled 21-nt oligomer (4R; see Fig. 2) and $\gamma$ - ${ }^{32}$ P-ATPlabeled small RNA isolated from a gel containing total RNA of the hemizygous transgenic tobacco line NLG4-He. carrying the DNA of the five GUS subclones. In Figure 5 , the phosphor image of the hybridization with the labeled small RNA fraction is shown. It demonstrates that the labeled small RNA fraction contains GUS-genederived sequences. Moreover, the hybridization is limited to the GUS subclones 2, 3, and 4. This confirms that in the GUS-silenced NLG4-He plants, the PTGSassociated small RNA is derived from the sequences present in the GUS2, GUS3, and GUS4 subclones.

\section{Fine mapping of the GUS sequences generating small RNA molecules}

To examine in more detail which parts of the GUS sequence generate PTGS-associated small RNA in NLG4$\mathrm{He}$, the labeled small RNA was used as a tool. Different double digests of GUS2, GUS3, and GUS4 subclone DNA were prepared with six- and four-base recognizing restriction enzymes. The DNA fragments were separated, blotted, and hybridized with the labeled small RNA fraction isolated from tobacco NLG4-He. Figure 6 shows the detailed maps (Fig. 6A) and phosphor image (Fig. 6B) of this hybridization. The hybridization with the small RNA probe is not uniform and fragments with stronger and weaker hybridization can be distinguished. Except for a few fragments in the GUS2 sequence, all sequences present in the subclones GUS2-4 show hybridization with the NLG4-He small 


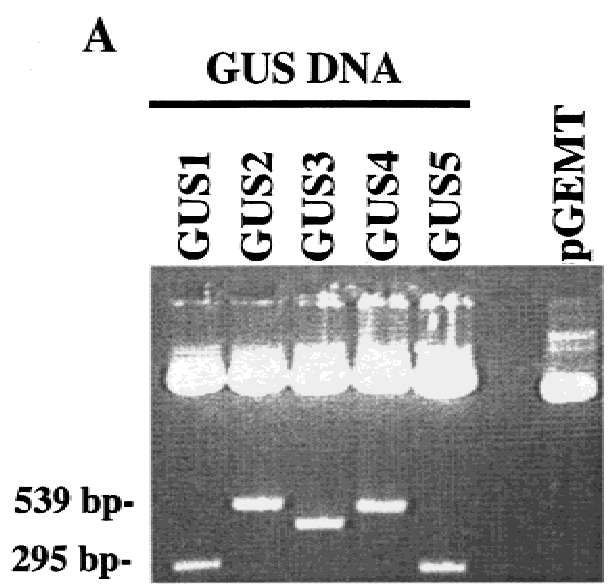

B

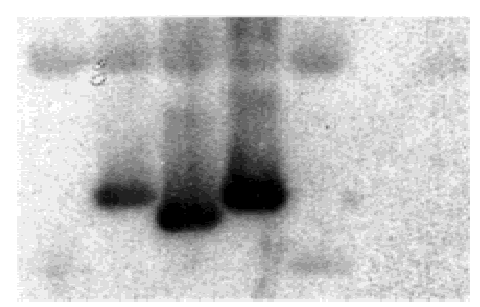

FIGURE 5. Mapping of the GUS regions represented in the small RNA fraction using labeled small RNA as a probe. A: Ethidiumbromidestained $1 \%$ agarose gel of plasmid DNA of the five GUS clones (see Fig. 2 for sizes and positions), digested with EcoRI. Empty pGEM-T Easy vector (pGEMT) is used as control. B: Phosphor image of the gel shown in $(\mathbf{A})$, after blotting and hybridization with the labeled small RNA from tobacco NLG4-He shown in Figure 4.

RNA probe. The difference is at the $5^{\prime}$ end of the GUS 2 subclone, where a 259-bp-long sequence did not hybridize with the labeled small RNA from NLG4-He (Fig. 6B). The error in this hybridization analysis is estimated to be of the size of an oligomer of about $15 \mathrm{nt}$ per hybridizing fragment. To confirm that the nonhybridizing fragments were present on the blot, the blot was stripped and rehybridized with a DNA probe covering the full sequence present in GUS2 to GUS4. Figure $6 \mathrm{C}$ shows that in the hybridization with this probe the 259-bp of the GUS2 sequence is visualized also. The hybridization analysis therefore establishes that a sequence of $554 \pm 15$ nt (= GUS1 + 259 nt from GUS2) does not generate GUS-homologous small RNA molecules. The rest of the 1,233 \pm 15 nt of the GUS mRNA is represented in the small RNA pool in the silenced tobacco line NLG4-He.

\section{Small RNA species in wild-type and nonsilenced plants}

In the isolation of the small RNA fraction from gel, the single-stranded oligomers used for size reference were visualized with the SYBR Green II RNA stain (Molecular Probes). In these experiments, we observed that the small RNA molecules could be visualized by this SYBR Green II stain also. This visualization allowed the analysis of wild-type and GUS-active tobacco RNA for the presence of such small RNA species. Figure 7A shows a SYBR Green II-stained gel with RNA isolated from wild-type tobacco, the GUS-active ANGLA13, and the GUS-silenced NLG4-He plants. Several discrete bands in the range of 21 and 30 nt are present in these RNA preparations. No obvious differences in SYBR Green II staining patterns between these three tobacco genotypes were detected in the small RNA area. This result shows that wild-type and nonsilenced transgenic tobacco plants contain small RNA fragments also. SYBR Green II staining as well as radioactive labeling with polynucleotide kinase allowed us to demonstrate a similar-sized small RNA fraction in wild-type Arabidopsis plants as well (results not shown).

\section{DISCUSSION}

An approximately 21-23-nt-short RNA species homologous to the silenced gene associates with PTGS in plants (Hamilton \& Baulcombe, 1999, 2000) and such a small RNA species may play an important role in the establishment and/or maintenance of PTGS. The original publication (Hamilton \& Baulcombe, 1999) involves elaborate isolation steps to detect the small RNA species. In this study we show that a standard and simpler method of total RNA isolation allows the easy and reproducible detection of small RNA species. The procedure used does not wash out or lose the small RNA. The RNA can be routinely visualized with mediumstringency hybridization using DNA probes. An additional advantage seems to be that there is less aspecific hybridization. We have compared the small RNA in the GUS-silenced tobacco line NLG4-He with wild-type tobacco and a GUS-active transgenic tobacco line using this simpler method of RNA isolation. This comparison confirmed the association of GUS-homologous small RNA with gene silencing as shown by Hamilton and Baulcombe (1999). On the basis of the observed hybridization intensities compared to a known amount of a GUS oligomer (Fig. 2), the amount of small GUS RNA is estimated to be about $100 \mathrm{ng}$ per $50 \mu \mathrm{g}$ NLG4-He RNA, thus, on the order of $0.2 \%$. Due to, among other factors, differences between RNA/DNA and DNA/DNA hybridization kinetics, this amount will be overestimated. A conservative estimate of the amount of the silencing-associated, GUS-homologous small RNA would be roughly $0.01-0.05 \%$ of the total RNA. The average total amount of mRNA in plants is estimated to be about $2 \%$ of the total RNA. The amount of GUS gene-homologous small RNA is therefore considerable. We could detect PTGS-associated small RNAs in both tobacco and Arabidopsis plants carrying different silenced (trans) genes equally easily. Therefore, it is not likely that tobacco line NLG4-He has exceptionally 
A

GUS2 $\quad$ GUS3

GUS4

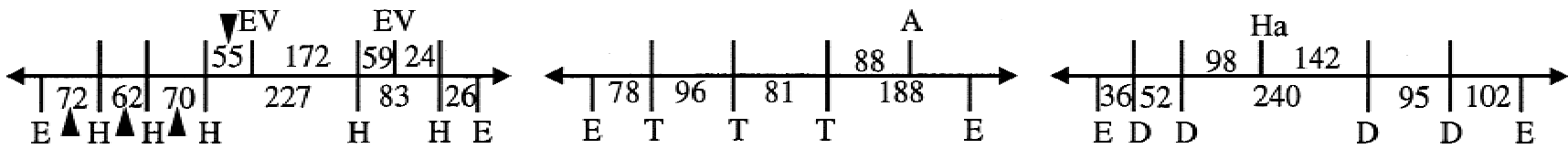

B

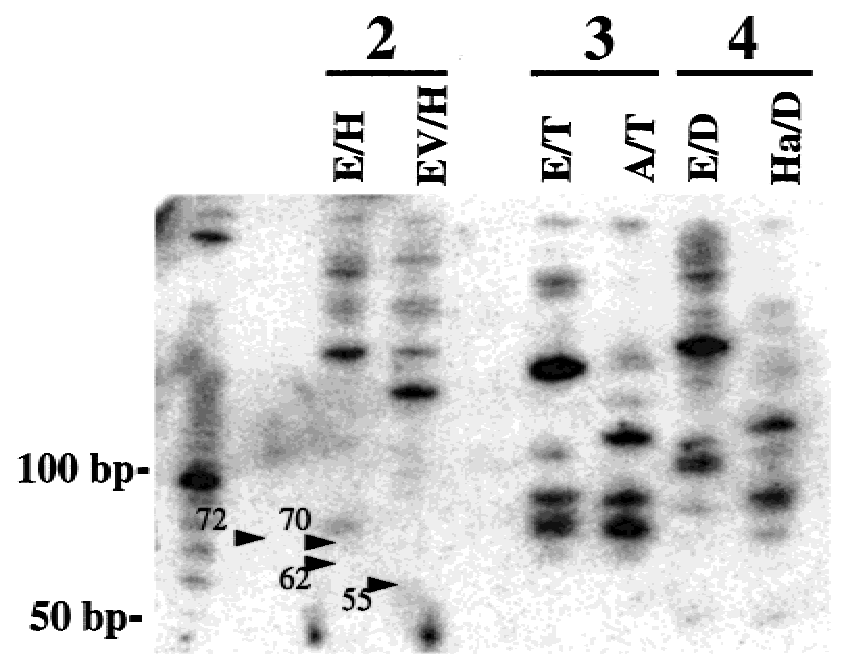

GUS subclones
FIGURE 6. Detailed fine mapping of the GUS regions that are represented in the small RNA fraction using labeled small RNA as a probe. A: Detailed restriction maps of the GUS subclones 2-4. Enzymes used: E: EcoRI, H: Hpall, EV: EcoRV, T: Taql, A: Alul, D: Dpnl, and Ha: Haell. Black triangles show the DNA fragments, which do not hybridize with the small RNA in (B), but with the GUS DNA in (C); these are the fragments in the GUS2 sequence found to be absent from the small RNA fraction. B: Phosphor image of a $6 \%$ polyacrylamide/8 M urea gel containing double digested GUS subclone plasmid DNA, after blotting and hybridization with the labeled small RNA isolated from tobacco NLG4-He (as in Fig. 4). C: Phosphor image of the blot shown in (B), after stripping and rehybridization with the full-length GUS sequence. high levels of PTGS-associated small RNA. The method described here is fast, reproducible, and sensitive. It facilitates further analysis of the occurrence and characteristics of the small RNA. Moreover, the ease of analysis will allow for screening larger populations of transgenic plants routinely for the presence of small RNA species. This will contribute to the early selection of stable, nonsilenced transgenic lines as well as easier identification of mutant lines impaired in accumulating small RNA.

A first question in the further analysis of the characteristics of PTGS-associated small RNA is what part of a silenced gene is generating the small RNA. The small RNA of tobacco line NLG4-He was characterized by hybridization using five GUS subclones as probes (Fig. 3). Further fine mapping of the small RNAgenerating sequences was performed with the help of gel-purified and labeled small RNA isolated from NLG4-He (Fig. 4) as a probe (Figs. 5 and 6). These analyses showed that the small RNA fraction is a mixture of similarly small-sized RNAs with different sequence contents, but all homologous to the GUS coding region. Apart from $554 \pm 15 \mathrm{nt}$ of the $5^{\prime}$ end (counted from the ATG), and the $3^{\prime}$ noncoding region, the remaining $1,233 \pm 15 \mathrm{bp}$ of the GUS coding region are generating small RNA species. This result suggests that the PTGS mechanism recognizes the continuous two-thirds of the $3^{\prime}$ end of the GUS coding region. In the hybridization with labeled small RNA (Fig. 6B), stronger and weaker hybridizing DNA fragments are observed. This could imply that different parts of the GUS mRNA generate small RNAs with different efficiencies, but the differences are more likely to reflect sequence differences in the 21-23-nt small RNA species.

The fine mapping of the small RNA generating sequences presented identifies a considerably larger sequence involved in generating of PTGS-associated small RNA than the sequence previously identified in the potato virus $X(P V X)$ system to be involved in GUS gene silencing (English et al., 1996). With the help of the PVX system, the GUS mRNA sequences that are targets for PTGS have been delimited to the $3^{\prime} 700 \mathrm{bp}$ of the GUS sequence. A 1.1-kb 5' part of the GUS gene was not an efficient target for GUS gene silencing. This 


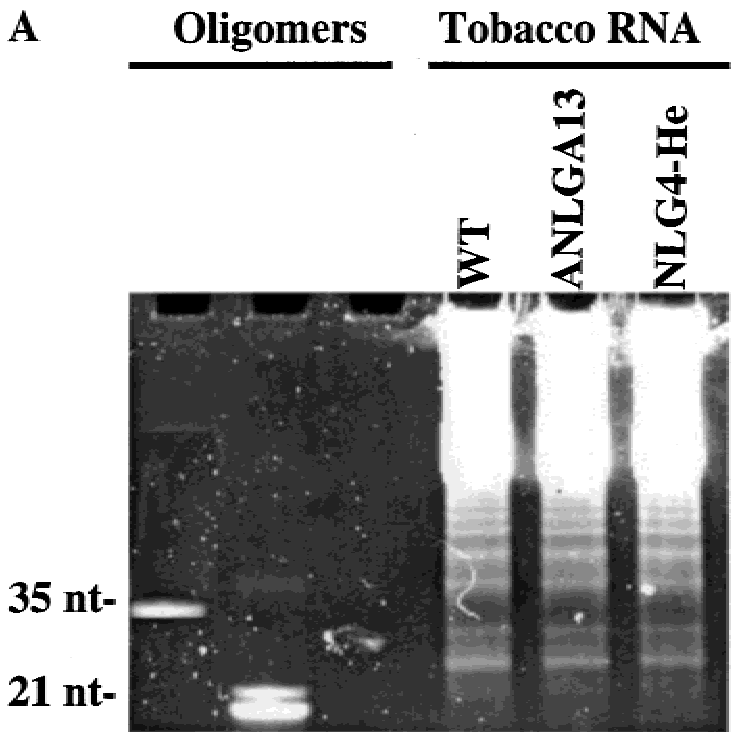

B

FIGURE 7. Visualization and labeling of the small RNA in GUS-active and wild-type tobacco compared to GUS-silenced tobacco. A: SYBR Green II-stained gel of $30 \mu \mathrm{g}$ RNA isolated from tobacco. WT: RNA from wild-type tobacco; NLG4-He: RNA from the hemizygous transgenic line NLG4-He, which is GUS silenced; ANGLA13: RNA from the homozygous transgenic line ANGLA13, which has GUS activity. As size markers, $150 \mathrm{ng}$ of two different oligomers were used. B: Phosphor image of a dried $17 \%$ polyacrylamide/8 M urea gel containing $\gamma_{-}{ }^{32} \mathrm{P}$-ATP-labeled small RNA isolated from wild-type tobacco RNA (WT). For comparison, labeled small RNA isolated from tobacco NLG4-He (as in Fig. 4) is run on the gel. As size markers, appropriate amounts of a $\gamma^{3}{ }^{33} \mathrm{P}$-ATP-labeled 21-mer oligo (4R, see Fig. 2) and the 10-bp ladder (Life Technologies) were used.

suggests that the sequences that are conferring GUS gene silencing in PVX do not fully overlap with the sequences that generate the silencing-associated small RNA fraction in plants. Possibly the GUS silencinginducing PVX viral system is not fully comparable with GUS silencing of transgenes in transgenic plants. Possibly the timing or kinetics of silencing in the viral system are different. Another explanation could be that the plants used for analysis in these two cases differ. The state and sensitivity of silencing in the GUS-silenced tobacco line NLG4-He may not be identical to that in the silenced tobacco line T4 used in the PVX analyses, a major difference being the promoter driving the GUS gene. These possibilities will require further analysis.

In the case of the gene encoding the Cowpea Mosaic Virus movement protein, silencing was shown to be directed against the distal part of the mRNA of the transgene (Sijen et al., 1996). Of the mature $\beta$-1,3glucanase mRNA sequence, the $5^{\prime}$ and the $3^{\prime}$ region containing the nontranslated regions proved to be inefficient silencing targets (Jacobs et al., 1999). In case of the phytoene desaturase gene, such a preference was not observed (Ruiz et al., 1998), whereas plants that exhibited PTGS for the coat protein of a sweet potato virus, different transgenic lines were shown to have different target specificities for the silenced transgene (Sonoda et al., 1999). This indicates that specificsequence characteristics, transgene configurations, and/or chromosomal positions may be important in defining the targets for gene silencing. The same is likely to be true for the sequences that generate PTGSassociated small RNAs. The NLG4-He tobacco line contains a complete inverted repeat of the GUS gene. In this tobacco, the proximal and the distal ends of the GUS RNA may be invisible for the small RNA-generating mechanism due to interaction with cap-binding proteins, poly A binding proteins, and/or ribosomes (Lee et al., 1997; Tanzer et al., 1997). The analyses developed here for tobacco line NLG4-He can now easily be performed on other silenced plant lines to show whether the generation of small RNA depends in part on the sequence characteristics and/or the chromosomal configuration or position of the silenced transgene.

The next questions are: how are the small RNAs generated and how do they fit in the mechanism of establishment and/or maintenance of the silenced state? Are they the signaling molecules that activate the silencing machinery, or are they the end products of the activated silencing machinery? Hamilton and Baulcombe (1999) suggested that the small RNA are the product of the RdRp that synthesizes RNA from an RNA template. A Neurospora quelling-defective strain is mutated in an RdRp enzyme (Cogoni \& Macino, 1999), showing the importance of this enzyme in fungal gene silencing. Arabidopsis mutants confirm the importance of this enzyme for PTGS (Dalmay et al., 2000b; Mourrain et al., 2000). The small RNAs may be the molecules that initiate the silencing. We here show that the PTGS-associated small RNA can be labeled by T4 polynucleotide kinase by the so-called forward reaction to 
generate a suitable probe (Fig. 4). No labeling was obtained for an RNA with a 5' P. This showed that the exchange reaction that can also be catalyzed by $\mathrm{T} 4$ polynucleotide kinase does not contribute to the labeling of small RNA under the experimental conditions used. The forward reaction requires that the $5^{\prime}$ end of the small RNA molecules not contain a phosphate group. Such a $5^{\prime}-\mathrm{OH}$ can be the result of endonucleases, but is not likely the result of any synthetic activity. The GUShomologous small RNA is therefore not likely to be a primary synthesis product of RdRp. Possibly RdRp synthesized larger RNA fragments that undergo degradation. Previous analyses of RNA degradation products in relation to gene silencing in plants have already involved endonuclease and exonuclease activities (Metzlaff et al., 1997; van Eldik et al., 1998; Litière et al., 1999).

Also, in other systems RNA degradation was involved. In a Drosophila in vitro system, injected dsRNA is degraded to 21-23 nt RNA species (Zamore et al., 2000). A partially purified nuclease fraction contained a similar small RNA species derived from the cyclin $\mathrm{E}$ transcript targeted by RNAi in Drosophila cells (Hammond et al., 2000). The C. elegans mut-7 gene, which is required for RNAi, possesses homology to an RNase (Ketting et al., 1999). Taken together, there are striking parallels between the mechanism of PTGS in plants and RNAi in other systems. If so, the small RNA is more likely to be a degradation end product derived from a larger precursor. The amount of injected dsRNA required to result in RNAi in $C$. elegans is very small (Fire et al., 1998). Therefore, the precursor may be a larger dsRNA molecule that initiates and maintains a self-perpetuating system resulting in considerable amounts of small RNA species. To elucidate the causal relationship between the initiation of silencing and the appearance of PTGS-related small RNA species would seem to require a well-defined window of time in which the gene silencing is initiated. The promoter driving the GUS gene in tobacco line NLG4 is light regulated and we are currently investigating if we can employ that promoter characteristic to define such a window of initiation of gene silencing in plants.

While visualizing the small RNA by SYBR Green II (Fig. 7A) and labeling (Fig. 7B), we were surprised to find that both in wild-type tobacco and Arabidopsis, a small RNA fraction is present of a size similar to the small RNA fraction in PTGS-silenced plants. This small RNA fraction from wild-type plants could also be isolated from a gel and labeled with T4 polynucleotide kinase. Although the small RNA fraction in wild-type plants may contain essentially random degradation products of the major ribosomal RNAs or other RNAs from plants, the fraction might include small RNAs derived from endogenous genes that are down-regulated by PTGS or a PTGS-like mechanism. The putative existence of small RNAs derived from endogenous genes would agree with the suggestion that PTGS is a part of a plant's basic repertoire to regulate gene expression. The possibility of isolating and labeling the small RNA from wild-type plants and using it as a probe will help to decide whether such endogenous genes being regulated by PTGS really do exist. If so, the approach will contribute to the identification and study of such gene(s).

\section{MATERIALS AND METHODS}

\section{Plant material}

The transgenic tobacco (Nicotiana tabacum L. cv Petit Havana SR1) lines used in this study were described previously (Nap et al., 1997). Transgenic tobacco line NLG4 contains a complex single locus of the light-regulated Lhca3.St.1promoter-GUS construct from the T-DNA vector pNLG. Tobacco line ANLGA13 contains an intact single copy of a similar GUS fusion in the T-DNA vector pANLGA. This line has a representative GUS activity (Mlynárová et al., 1994). Homogeneous hemizygous seed lots were obtained by backcrossing the homozygous transgenic line to wild-type. In addition, untransformed wild-type tobacco SR1 was used. Plants were germinated in soil and grown in a ventilated containment greenhouse.

\section{DNA manipulations}

All standard DNA manipulations, such as agarose gel electrophoresis, DNA cloning, PCR, plasmid technology, and Southern blotting, were performed essentially according to Sambrook et al. (1989), unless stated otherwise.

\section{Detection of gene silencing-related small RNA species by hybridization}

Plant total RNA was isolated with the TriZOL reagent (Life Technologies), essentially according to the manufacturer's recommendations. In short, leaf material was harvested in liquid nitrogen and ground to a powder. Approximately 100 to $300 \mathrm{mg}$ (one-third of an Eppendorf tube) was used to add $1 \mathrm{~mL}$ TriZOL reagens. This mixture was mixed well, after which $200 \mu \mathrm{L}$ chloroform was added. This was shaken well again, centrifuged $\left(12.000 \times g, 15 \mathrm{~min}, 4^{\circ} \mathrm{C}\right)$ and the RNA in the supernatant (approximately $700 \mu \mathrm{L}$ ) was precipitated with $500 \mu \mathrm{L}$ isopropanol. After a short $70 \%$ ethanol wash, the RNA was dissolved in $30 \mu \mathrm{L}$ of sterile distilled water. The RNA concentration was determined spectrophotometrically and RNA integrity was routinely checked on a nondenaturing $1 \%$ agarose gel. Separation of total RNA was performed in a $6 \%$ polyacrylamide/8 M urea gel using the $6 \%$ Sequagel mix (BIOzym) in the Protean apparatus (BioRad). Samples were denatured for $10 \mathrm{~min}$ at $65^{\circ} \mathrm{C}$ in $1 \times$ loading dye $(2 \times$ loading dye $=98 \%$ deionized formamide, $10 \mathrm{mM}$ EDTA $(\mathrm{pH}=8.0)$, $1 \mathrm{mg} / \mathrm{mL}$ xylene cyanol FF, and $1 \mathrm{mg} / \mathrm{mL}$ bromophenol blue). After separation, RNA was electroblotted in $50 \mathrm{mM}$ Tris-borateEDTA buffer ( $\mathrm{pH}=8.0$ ) onto a Hybond- $\mathrm{N}^{+}$membrane (Amersham). Transfer was done overnight at room temperature at 20 V (Stoeckle \& Guan, 1993), after which RNA was fixed on the membrane using a UV Crosslinker (Stratagene). A radio- 
actively labeled DNA probe was prepared with the Megaprime random priming kit (Amersham) according to the recommendations of the manufacturer. Prehybridization of the membranes was performed for at least $2 \mathrm{~h}$ at $50^{\circ} \mathrm{C}$ in a hybridization solution (10\% Dextran sulfate, 1\% SDS, $1 \mathrm{M}$ $\mathrm{NaCl}, 50 \mathrm{mM}$ Tris-HCL, $\mathrm{pH}=7.5,80 \mu \mathrm{g} / \mathrm{mL}$ denatured salmon sperm DNA), using $25 \mathrm{~mL}$ of solution per blot. For hybridization, the probe was denatured by boiling for $10 \mathrm{~min}$ in the presence of $4 \mathrm{mg}$ of sonicated salmon sperm DNA for each $25 \mathrm{~mL}$ of (pre)hybridization solution and immediately added to the prehybridization mixture. Hybridization was performed overnight at $50^{\circ} \mathrm{C}$. After hybridization, a membrane was washed in $2 \times \mathrm{SSC}$ at $50^{\circ} \mathrm{C}$ for $5 \mathrm{~min}$, followed by several washing steps in $2 \times \mathrm{SSC}, 1 \% \mathrm{SDS}$ at $50^{\circ} \mathrm{C}$ for $20 \mathrm{~min}$. Hybridization signals were visualized by autoradiography on Fuji 100NIF films or on a Fuji B2000 Phosphorlmager with BasReader and TINA software (Raytest).

\section{Visualization and isolation of small RNA}

To visualize small RNA without hybridization, RNA was separated on a $17 \%$ polyacrylamide/8 M urea gel prepared from a 37.5:1 acrylamide/bisacrylamide solution. Following electrophoresis, the RNA was stained by shaking for 30-40 min with $30 \mathrm{~mL}$ of a 1:10,000 dilution in $1 \times$ TBE of the SYBR Green II RNA gel stain (Molecular Probes). The RNA was visualized with a Lumi-Imager (Roche) using the LumiAnalyst software for this stain. As size markers, oligomers of known size (Eurogentec) were used. The small RNA fraction (20-35 bp) was recovered from the gel with a "crush-and-soak" method (Faulkner-Jones, 1995). The relevant polyacrylamide gel piece was cut from the gel and eluted in $500 \mu \mathrm{L}$ elution buffer ( $80 \%$ formamide, $40 \mathrm{mM}$ PIPES, $\mathrm{pH}=6.4,1 \mathrm{mM}$ EDTA, $400 \mathrm{mM} \mathrm{NaCl}$ ) at $37^{\circ} \mathrm{C}$ overnight with gentle shaking. The eluted RNA was precipitated with 1 vol of isopropanol at room temperature for $30 \mathrm{~min}$ and the RNAs were recovered by centrifugation. The pellet was washed with $70 \%$ ethanol and resuspended in $200 \mu \mathrm{L}$ of $10 \mathrm{mM}$ DTT. RNA was reprecipitated by adding $0.1 \mathrm{vol}$ of $8 \mathrm{M} \mathrm{LiCl}$ and an equal volume of isopropanol at room temperature for $30 \mathrm{~min}$. The pellet was shortly washed with $70 \%$ ethanol and resuspended in $20 \mu \mathrm{L}$ of sterile distilled water.

\section{Labeling of small RNA and DNA oligomers}

One-third (6 $\mu \mathrm{L})$ of the gel-isolated small RNA was radioactively labeled with the forward reaction of the Kinase Max 5 '-End-Labeling Kit (Ambion) according to the manufacturer's manual, using $\gamma^{3}{ }^{32} \mathrm{P}$-ATP $(7,000 \mathrm{Ci} / \mathrm{mmol} ; 150 \mathrm{mCi} / \mathrm{mL}$; ICN). Part of the labeled RNA (about 1/1,000th of the total) was routinely analyzed on a $17 \%$ polyacrylamide/8 $\mathrm{M}$ urea gel as described above. DNA oligomers and the 10-bp DNA ladder (Life Technologies) were labeled with the same kit, using $\gamma^{-33}$ P-ATP $(3,000 \mathrm{Ci} / \mathrm{mmol} ; 10 \mathrm{mCi} / \mathrm{mL} ; \mathrm{ICN})$.

\section{ACKNOWLEDGMENTS}

We wish to thank Dick Geurtsen of the Plant Research International's greenhouse team for plant care; Maarten van der Heijden for the GUS subclones and Andrea Tóth (Gödöllö, Hungary), Andy Pereira (Plant Research International,
Wageningen) and an anonymous reviewer for helpful comments on earlier versions of this manuscript. The investigations were supported by a visiting scientist fellowship from the Dutch Ministry of Agriculture, Nature Management and Fisheries (to G.H.) as well as by an Eötvös fellowship of the Hungarian Academy of Sciences (to G.H.), by a program subsidy of the Life Sciences Foundation of the Dutch Organisation for Scientific Research (to L.M.) and by programme subsidy 354 from the Dutch Ministry of Agriculture, Nature Management and Fisheries.

Received June 8, 2000; returned for revision

June 29, 2000; revised manuscript received July 25, 2000

\section{REFERENCES}

Baulcombe DC. 1999. Fast forward genetics based on virus-induced gene silencing. Curr Opin Plant Biol 2:109-113.

Baulcombe DC, English JJ. 1996. Ectopic pairing of homologous DNA and post-transcriptional gene silencing in transgenic plants. Curr Opin Biotechnol 7:173-180.

Bosher JM, Labouesse M. 2000. RNA interference: Genetic wand and genetic watchdog. Nature Cell Bio 2:E31-E36.

Chuang C-F, Meyerowitz EM 2000. Specific and heritable genetic interference by double-stranded RNA in Arabidiopsis thaliana. Proc Natl Acad Sci USA 97:4985-4990.

Cogoni C, Macino G. 1997. Isolation of quelling-defective (qde) mutants impaired in posttranscriptional transgene-induced gene silencing in Neurospora crassa. Proc Natl Acad Sci USA 94:1023310238.

Cogoni C, Macino G. 1999. Gene silencing in Neurospora crassa requires a protein homologous to RNA-dependent RNA polymerase. Nature 399:166-169.

Dalmay T, Hamilton A, Mueller E, Baulcombe DC. 2000a. Potato Virus $\mathrm{X}$ amplicons in Arabidopsis mediate genetic and epigenetic gene silencing. Plant Cell 12:369-380.

Dalmay T, Hamilton A, Rudd S, Angell S, Baulcombe DC. 2000b. An RNA-dependent RNA polymerase gene in Arabidopsis is required for posttranscriptional gene silencing mediated by a transgene but not by a virus. Cell 101:543-553.

English JJ, Mueller E, Baulcombe DC. 1996. Suppression of virus accumulation in transgenic plants exhibiting silencing of nuclear genes. Plant Cell 8:179-188.

Faulkner-Jones B. 1995. Recovery of RNA fragments from polyacrylamide gels. http://grimwade.biochem.unimelb.edu.au/bfjones/ gen7/m7a4.htm.

Fire A, Xu S, Montgomery MK, Kotas SA, Driver SE, Mello CC. 1998. Potent and specific genetic interference by double-stranded RNA in Caenorhabditis elegans. Nature 391:806-811.

Hamilton AJ, Baulcombe DC. 1999. A species of small antisense RNA in posttranscriptional gene silencing in plants. Science 286:950-952.

Hamilton AJ, Baulcombe DC. 2000. A class of short RNA specific to post-transcriptional gene silencing (PTGS): Possible specificity determinants and signal molecules. Poster S07-16, 6th International Congress of Plant Molecular Biology, ISPMB, Québec, Canada, June 18-24.

Hammond SM, Bernstein E, Beach D, Hannon GJ. 2000. An RNAdirected nuclease mediates post-transcriptional gene silencing in Drosophila cells. Nature 404:293-296.

Jacobs JJ, Sanders M, Bots M, Andriessen M, Van Eldik GJ, Litière K, Van Montagu M, Cornelissen M. 1999. Sequences throughout the basic beta-1,3-glucanase mRNA coding region are targets for homology dependent post-transcriptional gene silencing. Plant $J$ 20:143-152.

Ketting RF, Haverkamp TH, van Luenen HG, Plasterk RH. 1999. Mut-7 of $C$. elegans, required for transposon silencing and RNA interference, is a homolog of Werner syndrome helicase and RNaseD. Cell 99:133-141.

Ketting RF, Plasterk RHA. 2000. A genetic link between co-suppression and RNA interference in C. elegans. Nature 404:296-298. 
Lee KY, Baden C, Howie WJ, Bedbrook J, Dunsmuir P. 1997. Posttranscriptional gene silencing of ACC synthase in tomato results from cytoplasmatic RNA degradation. Plant J 12:1127-1137.

Lindbo JA, Silva-Rosales L, Proebsting WM, Dougherty WG. 1993. Induction of a highly specific antiviral state in transgenic plants: Implications for regulation of gene expression and virus resistance. Plant Cell 5:1749-1759.

Litière K, van Eldik GJ, Jacobs JJ, Van Montagu M, Cornelissen M. 1999. Posttranscriptional gene silencing of gn1 in tobacco triggers accumulation of truncated gn1-derived RNA species. RNA 5:1364-1373.

Metzlaff M, O'Dell M, Cluster PD, Flavell RB. 1997. RNA-mediated RNA degradation and chalcone synthase A silencing in petunia. Cell 88:845-854.

Misquitta L, Paterson BM. 1999. Targeted disruption of gene function in Drosophila by RNA interference (RNA-i): A role for nautilus in embryonic somatic muscle formation. Proc Natl Acad Sci USA 96:1451-1456.

Mlynárová L, Loonen A, Heldens J, Jansen RC, Keizer P, Stiekema WJ, Nap JP. 1994. Reduced position effect in mature transgenic plants conferred by the chicken lysozyme matrix-associated region. Plant Cell 6:417-428.

Montgomery MK, Xu S, Fire A. 1998. RNA as a target of doublestranded RNA-mediated genetic interference in Caenorhabditis elegans. Proc Natl Acad Sci USA 95:15502-15507.

Mourrain P, Béclin C, Elmayan T, Feuerbach F, Godon C, Morel JB, Jouette D, Lacombe AM, Nikic S, Picault N, Rémoué K, Sanial M, Vo TA, Vaucheret H. 2000. Arabidopsis sgs2 and sgs3 genes are required for posttranscriptional gene silencing and natural virus resistance. Cell 101:533-542.

Nap JP, Conner AJ, Mlynárová L, Stiekema WJ, Jansen RC. 1997. Dissection of a synthesized quantitative trait to characterize transgene interactions. Genetics 147:315-320.

Ngo H, Tschudi C, Gull K, Ullu E. 1998. Double-stranded RNA induces mRNA degradation in Trypanosoma brucei. Proc Natl Acad Sci USA 95:14687-14692.
Ruiz MT, Voinnet O, Baulcombe DC. 1998. Initiation and maintenance of virus-induced gene silencing. Plant Cell 10:937-946.

Sambrook J, Fritsch EF, Maniatis T. 1989. Molecular cloning: A laboratory manual. Cold Spring Harbor, New York: Cold Spring Harbor Laboratory Press.

Sanchez Alvarado A, Newmark PA. 1999. Double-stranded RNA specifically disrupts gene expression during planarian regeneration. Proc Natl Acad Sci 96:5049-5054.

Sharp PA. 1999. RNAi and double-strand RNA. Genes \& Dev 13: $139-141$.

Sijen T, Wellink J, Hiriart J-B, van Kammen A. 1996. RNA-mediated virus resistance: Role of repeated transgenes and delineation of targeted regions. Plant Cell 8:2277-2294.

Sonoda S, Mori M, Nishiguchi M. 1999. Homology-dependent virus resistance in transgenic plants with the coat protein gene of sweet potato feathery mottle potyvirus: Target specificity and transgene methylation. Phytopath 89:385-391.

Stoeckle MY, Guan L. 1993. Improved resolution and sensitivity of northern blots using polyacrylamide-urea gels. BioTechniques 15:230-231.

Tanzer MM, Thompson WF, Law MD, Wernsman EA, Uknes S. 1997. Characterization of posttranscriptionally suppressed transgene expression that confers resistance to tobacco etch virus infection in tobacco. Plant Cell 9:1411-1423.

van Eldik GJ, Litière K, Jacobs JJ, Van Montagu M, Cornelissen M. 1998. Silencing of beta-1,3-glucanase genes in tobacco correlates with an increased abundance of RNA degradation intermediates. Nucleic Acids Res 26:5176-5181.

Wassenegger M, Pélissier T. 1998. A model for RNA-mediated gene silencing in higher plants. Plant Mol Biol 37:349-362.

Wianny F, Zernicka-Goetz M. 2000. Specific interference with gene function by double stranded RNA in early mouse development. Nature Cell Biol 2:70-75.

Zamore PD, Tuschl T, Sharp PA, Bartel DP. 2000. RNAi: Doublestranded RNA directs the ATP-dependent cleavage of mRNA at 21 to 23 nucleotide intervals. Cell 101:25-33. 